\title{
ON A CLASS OF COUNTABLY PARACOMPACT SPACES
}

\author{
JOHN MACK
}

In this note we shall characterize a topological property which is stronger than countable paracompactness but which is equivalent to it for normal spaces.

A real valued function on a topological space $X$ is locally bounded if each point has a neighborhood on which the function is bounded. Let $C(X)$ denote the set of real valued continuous functions on $X$. A topological space is a $c b$-space if for each locally bounded function $h$, there exists $f \in C(X)$ such that $f \geqq|h|$. J. G. Horne, Jr. initiated a study of $c b$-spaces and reported on his work in [2].

For $f \in C(X)$, the set on which $f$ vanishes is called the zero-set of $f$ and it is denoted by $Z(f)$. The complement of a zero-set is called a cozero-set. A cozero cover is a cover consisting of cozero-sets. A family of continuous functions is locally finite if the collection of cozero-sets associated with the family is a locally finite collection of sets. A family $\mathcal{F}$ of continuous functions is a partition of unity if $0 \leqq f$ for all $f \in \mathcal{F}$ and $\sum_{f \in \mathcal{F}} f(x)=1$ for all $x \in X$. A partition of unity is subordinate to a cover if the collection of cozero-sets associated with the partition is a refinement of the cover. A countable cover $\left\{U_{n}\right\}$ is an increasing cover if $U_{n} \subset U_{n+1}$ for all $n$. In this paper the term cover will be used to mean open cover.

Theorem 1. For any topological space $X$, the following statements are equivalent:

(a) $X$ is a cb-space.

(b) Given an upper-semicontinuous function $h$ on $X$, there exists $f \in C(X)$ such that $f \geqq h$.

(c) Given a positive (nonvanishing) lower-semicontinuous function $g$ on $X$, there exists $f \in C(X)$ such that $0<f(x) \leqq g(x)$ for all $x \in X$.

(d) For each countable increasing cover of $X$, there exists a locally finite partition of unity subordinate to that cover.

(e) For each countable increasing cover of $X$, there exists a partition of unity subordinate to that cover.

(f) Each countable increasing cover of $X$ has a locally finite cozero refinement.

(g) Each countable increasing cover of $X$ has a $\sigma$-locally finite cozero refinement. 1964.

Presented to the Society, January 27, 1965; received by the editors February 10 , 
(h) Each countable increasing cover of $X$ has a countable cozero refinement.

(i) Given a decreasing sequence $\left\{F_{n}\right\}$ of closed sets in $X$ with empty intersection, there exists a sequence $\left\{Z_{n}\right\}$ of zero-sets with empty intersection such that $Z_{n} \supset F_{n}$.

Proof. (a) $\rightarrow$ (b). If $h$ is upper-semicontinuous, then $h^{+}$is locally bounded. Thus there exists $f \in C(X)$ such that $f \geqq h^{+} \geqq h$.

(b) $\rightarrow$ (c). If $g$ is strictly positive and lower-semicontinuous, then $g^{-1}$ exists and is upper-semicontinuous. Let $\phi \in C(X)$ be such that $\phi \geqq g^{-1}$. Then $f=\phi^{-1} \in C(X)$ and $0<f(x) \leqq g(x)$ for all $x \in X$.

$\left(\right.$ c) $\rightarrow$ (d). For a countable increasing cover $\left\{U_{n}\right\}$, define $g(x)=1$ on $U_{1}$ and $g(x)=n^{-1}$ on $U_{n}-U_{n-1}$ for $n>1$. Then $g$ is lower-semicontinuous and strictly positive. For $f \in C(X)$ such that $0<f(x)$ $\leqq g(x)$ on $X$, define $\phi_{n}=[(n+1) f-1]+[(n-1) f-1]-$ and $\phi=\sum \phi_{n}$ (here $1(x)=1$ for all $x \in X)$. Since $\left\{\phi_{n}\right\}$ is locally finite, $\phi \in C(X)$. Furthermore, $\phi$ is nonvanishing; thus $\phi$ is a unit of the ring $C(X)$. It now follows that $\left\{\phi^{-1} \phi_{n}\right\}$ is a locally finite partition of unity. That this partition is subordinate to $\left\{U_{n}\right\}$ is a consequence of the following: $X-Z\left(\phi^{-1} \phi_{n}\right)=\left\{x:(n+1)^{-1}<f(x)<(n-1)^{-1}\right\}$ $C\left\{x:(n+1)^{-1}<g(x)\right\}=U_{n}$.

Statements (d), (e), and (f) are equivalent [6, 1.2]. Clearly (f) implies (g).

$(\mathrm{g}) \rightarrow(\mathrm{h})$. Let $\mho$ be a $\sigma$-locally finite cozero refinement of the increasing cover $\left\{U_{n}\right\}$. Then $V=U v_{m}$ where each $\mho_{m}$ is a locally finite collection of cozero-sets. Let $V_{m n}$ denote the union of the sets $V$ in $\vartheta_{m}$ such that $V \subset U_{n}$. Since $V_{m n}$ is the union of a locally finite collection of cozero-sets, it is a cozero-set. Thus $\left\{V_{m n}\right\}$ is a countable cozero refinement of $\left\{U_{n}\right\}$.

$(\mathrm{h}) \rightarrow(\mathrm{i})$. If $\left\{F_{n}\right\}$ is a decreasing sequence of closed sets for which $\cap F_{n}$ is empty, then $\left\{X-F_{n}\right\}$ is an increasing cover of $X$. Let $A$ be a countable subset of $C(X)$ such that $\{X-Z(f)\}_{f \in A}$ is a refinement of $\left\{X-F_{n}\right\}$. Denote by $Z_{n}$ the intersection of those $Z(f), f \in A$ for which $Z(f) \supset F_{n}$. Each $Z_{n}$ is a countable intersection of zero-sets and hence is a zero-set $[3, \S 1.14]$. Now $\cap Z_{n}=\bigcap_{f \in A} Z(f)$ is vacuous. Whence $\left\{Z_{n}\right\}$ is the desired sequence.

(i) $\rightarrow$ (a). Let $h$ be a locally bounded function on $X$ and define $F_{n}=\operatorname{cl}\{x:|h(x)| \geqq n\}$. Then $\left\{F_{n}\right\}$ is a decreasing sequence of closed sets. Since $h$ is locally bounded, this sequence has empty intersection. Thus by (i) there exists a sequence $\left\{g_{n}\right\}$ in $C(X)$ such that $Z\left(g_{n}\right) \supset F_{n}$ and $\cap Z\left(g_{n}\right)$ is empty. Define $f_{n}=1-\bigvee_{i \leq n} n\left|g_{i}\right| \wedge 1$ where $1(x)=1$ for all $x \in X$. Given $x \in X$, there exists $i$ such that $g_{i}(x) \neq 0$; whence there 
exists a positive integer $j$ such that $\left|g_{i}(x)\right|>j^{-1}$. If $n \geqq i$ and $n \geqq j$, then $n\left|g_{i}(y)\right| \geqq j\left|g_{i}(y)\right|>1$ on a neighborhood of $x$. Thus $f_{n}$ vanishes on that neighborhood provided $n \geqq \max \{i, j\}$. This shows that $\left\{f_{n}\right\}$ is locally finite. Therefore $f=1+\sum f_{n}$ is an element of $C(X)$. On $F_{n}$, we have $g_{i}(x)=0$ and $f_{i}(x)=1$ for $i \leqq n$. Thus $f(x) \geqq n+1>|h(x)|$ on $F_{n}-F_{n+1}$. This proves that $f \geqq|h|$.

REMARK 1. It should be noted that the zero-sets play the same role in the above characterization that the closed $G_{\delta}$-sets play in Dowker's characterization [1] of normal and countably paracompact spaces. This is not surprising since in normal spaces the zero-sets are precisely the closed $G_{\delta}$-sets.

REMARK 2 . In a completely regular space, the cozero-sets form a base for the topology. Hence, in such spaces, every cover has a cozero refinement. This fact emphasizes the significance of the local finiteness and countability requirements in (f) and $(h)$.

REMARK 3. If the word "increasing" is deleted from statements (d) through ( $h$ ), and "decreasing" is deleted from (i), then each becomes a characterization of normal and countably paracompact spaces. $[6,1.1$ and 1.2] or [7].

We are now able to state the following corollaries. Corollary 2 was originally proved by Horne [2].

Corollary 2. (i) A cb-space is countably paracompact.

(ii) A normal and countably paracompact space is a cb-space.

Corollary 3. A countably compact space is a cb-space.

Corollary 4. A closed subspace of a cb-space is a cb-space.

A subset $A$ of a topological space $X$ is called a generalized cozeroset (generalized $F_{\sigma}$-set) if each open set containing $A$ contains a cozeroset (respectively, $F_{\sigma}$-set) containing $A$.

Lemma 5. In a normal space, a set is a generalized cozero-set if and only if it is a generalized $F_{\sigma}$-set.

Proof. Since a cozero-set is an $F_{\sigma}$-set, the "only if" part is trivial. To prove the "if" part, it suffices to show that in a normal space each $F_{\sigma}$-set is a generalized cozero-set. Let $A=U F_{n}$ where each $F_{n}$ is closed. Given an open set $G \supset A$, there exists a sequence $\left\{Z_{n}\right\}$ of zero-sets such that $F_{n} \subset X-Z_{n} \subset G$. If $Z=\cap Z_{n}$, then $Z$ is a zero-set and $A \subset X-Z \subset G$. Thus $A$ is a generalized cozero-set.

THEOREM 6. Each generalized cozero-subspace of a cb-space is a cbspace. 
Proof. First, we shall consider a special case. Let $X$ be a $c b$-space and $Y$ be a cozero-set in $X$. Then there exists $g \in C(X), 0 \leqq g$ such that $Y=X-Z(g)$. Set $F_{n}=\left\{x: n^{-1} \leqq g(x)\right\}$. Given an upper-semicontinuous function $h$ defined on $Y$, set $h_{n}(x)=h^{+}(x)$ on $F_{n}$ and $h_{n}(x)=0$ on $X-F_{n}$. Then each $h_{n}$ is an upper-semicontinuous function on $X$; whence there exist $f_{n} \in C(X)$ such that $h_{n} \leqq f_{n}$. Also, for $n>2$ there exist $g_{n} \in C(X), g_{n} \geqq 0$ such that $Z\left(g_{n}\right) \supset F_{n-2}$ and $g_{n}(x)=1$ on $X-F_{n-1}$ (appropriate modifications of $g$ will give such $g_{n}$ ). Set $g_{1}=g_{2}=1$ and define $f=\bigvee_{n}\left(f_{n} g_{n} \mid Y\right)$. Since $\left\{g_{n} \mid Y\right\}$ is a locally finite family in $C(Y), f$ is an element of $C(Y)$. On $F_{n}-F_{n-1}, g_{n}(x)=1$ and $h^{+}(x)=h_{n}(x) \leqq f_{n}(x) \leqq f(x)$. Therefore $h \leqq f$. This proves the special case. The theorem follows by means of part (f) of Theorem 1, from the special case just proved.

The following previously known ([5] or [6, Corollary 1.6]) result is an immediate consequence of Lemma 5 and Theorem 6.

CoRollary 7. Each generalized $F_{\sigma}$-subspace of a normal and countably paracompact space is normal and countably paracompact.

As noted in [2], a product of $c b$-spaces may fail to be $c b$ or even countably paracompact. Also, the product of a normal and countably compact space with a compact space may fail to be normal [3, 8M.4]. The following theorem displays a property of $c b$-spaces which is not possessed by normal and countably paracompact spaces.

THEOREM 8. The product of a cb-space and a locally compact, paracompact (Hausdorff) space is a cb-space.

Proof. First, consider the special case where $Y$ is compact. Let $\left\{F_{n}\right\}$ be a decreasing sequence of closed sets in $X \times Y$ with empty intersection and let $A_{n}$ be the projection of $F_{n}$ into $X$. Since $Y$ is compact, the projection of $X \times Y$ onto $X$ is a closed mapping. Hence $\left\{A_{n}\right\}$ is a decreasing sequence of closed sets in $X$. Since, for each $x \in X$, the set $\{x\} \times Y$ is compact, this set fails to meet some $F_{n}$. Therefore $\left\{A_{n}\right\}$ has empty intersection. According to Theorem 1, there exists a sequence $\left\{Z_{n}\right\}$ of zero-sets in $X$ with empty intersection such that $Z_{n} \supset A_{n}$. Then $\left\{Z_{n} \times Y\right\}$ is a sequence of zero-sets in $X \times Y$ with empty intersection such that $Z_{n} \times Y \supset F_{n}$. This proves the special case. Next, let $Y$ be locally compact and paracompact. Then there exists a locally finite cover $\mathcal{u}$ of $Y$ consisting of relatively compact sets. Since $Y$ is normal, there exists a mapping $F$ from $\mathfrak{U}$ to the topology on $Y$ such that $\operatorname{cl} F(U) \subset U$ and $\{F(U): U \in \mathcal{U}\}$ covers $Y$. Let $h$ be a locally bounded function on $X \times Y$. By the special case just proved $X \times \operatorname{cl} U$ is a $c b$-space. Thus, since $X \times F(U)$ and 
$X \times(Y-U)$ are completely separated, it follows that there exists $f_{U} \in C(X \times Y)$ for which $f_{U}(x, y) \geqq|h(x, y)|$ on $X \times F(U)$ while $f_{U}$ vanishes outside $X \times U$. Since $\mathcal{u}$ is locally finite, $f=V_{U \in \mathcal{U}} f_{U}$ exists in $C(X \times Y)$. Clearly, $f \geqq|h|$. This proves that $X \times Y$ is a $c b$-space.

We shall now show that for certain non-normal spaces the $c b$ property is equivalent to countable paracompactness. A topological space $X$ is pseudocompact if every real valued continuous function on $X$ is bounded.

Theorem 9. Let $X$ be a completely regular pseudocompact space. Then the following statements are equivalent:

(i) $X$ is countably compact.

(ii) $X$ is a cb-space.

(iii) $X$ is countably paracompact.

Proof. The implications (i) $\rightarrow$ (ii) and (ii) $\rightarrow$ (iii) follow from Corollaries 2 and 3 . We shall prove that (iii) $\rightarrow$ (i). Suppose $X$ is countably paracompact but not countably compact. Then there is a countably infinite set $F=\left\{x_{1}, x_{2}, \cdots, x_{n}, \cdots\right\}$ which has no limit points. For each $n$, let $U_{n}$ be an open set such that $F \cap U_{n}=\left\{x_{n}\right\}$. Then $\left\{U_{n}\right\} \cup\{X-F\}$ is a cover of $X$. This cover has a locally finite refinement $\left\{V_{n}\right\} \cup\{X-F\}$ such that $V_{n} \subset U_{n}$. Then $x_{n} \in V_{n}$. Let $f_{n} \in C(X)$ be such that $f_{n}$ vanishes on $X-V_{n}$ while $f_{n}\left(x_{n}\right)=n$. Since $\left\{V_{n}\right\}$ is locally finite, $f=\mathrm{V} f_{n}$ exists in $C(X)$. Clearly, $f$ is unbounded. This is a contradiction since $X$ is pseudocompact.

REMARK. Theorem 9 generalizes to non-normal spaces the part of Theorem 1.8 in [6] for which $m=\boldsymbol{N}_{0}$.

Theorem 10. Let $X$ be a topological space. Then the following statements are equivalent.

(i) $X$ is countably paracompact.

(ii) For each increasing cover $\left\{U_{n}\right\}$ of $X$, there exists a refinement $\left\{V_{n}\right\}$ such that $\operatorname{cl} V_{n} \subset U_{n}$.

(iii) For each locally bounded function $h$ defined on $X$, there exists a locally bounded lower-semicontinuous function g such that $|h| \leqq g$.

Proof. The equivalence of (i) and the closed set dual of (ii) is proved in [4].

(ii) $\rightarrow$ (iii). Let $h$ be locally bounded and set

$$
U_{n}=\operatorname{int}\{x:|h(x)| \leqq n\} .
$$

Then $\left\{U_{n}\right\}$ is an increasing cover for $X$. Let $\left\{V_{n}\right\}$ be a refinement such that $\mathrm{cl} V_{n} \subset U_{n}$ and define $g(x)=\sup \left\{n: x \notin \bigcup_{k<n} \operatorname{cl} V_{k}\right\}$. Then $g$ is lower-semicontinuous and $g(x) \leqq n$ on $V_{n}$. Thus $g$ is locally 
bounded. If $g(x)=n$, then $x \in \operatorname{cl} V_{n} \subset U_{n}$; whence $|h(x)| \leqq n$. Therefore $|h| \leqq g$.

(iii) $\rightarrow$ (i). It suffices to show that (iii) implies that each increasing cover of $X$ has a locally finite refinement. Let $\left\{U_{n}\right\}$ be an increasing cover of $X$ and define $h(x)=\inf \left\{n: x \in U_{n}\right\}$. Then $h$ is locally bounded since it is positive and upper-semicontinuous. Let $g$ be a locally bounded, lower-semicontinuous function such that $g \geqq h$. Set $V_{n}=\{x: n-1<g(x)\} \cap\{x: h(x)<n+1\}$. Then $\left\{V_{n}\right\}$ is a refinement of $\left\{U_{n}\right\}$. Also, $\left\{V_{n}\right\}$ is locally finite since $g$ is locally bounded.

A topological space is extremally disconnected if every open set has an open closure.

THEOREM 11. Let $X$ be an extremally disconnected space. Then $X$ is a cb-space if and only if it is countably paracompact.

Proof. We need prove only the "if" part. Suppose $X$ is countably paracompact and let $\left\{U_{n}\right\}$ be an increasing cover of $X$. Then there exists a refinement $\left\{V_{n}\right\}$ such that $\operatorname{cl} V_{n} \subset U_{n}$. Since $X$ is extremally disconnected, $\operatorname{cl} V_{n}$ is open. Therefore $\left\{\operatorname{cl} V_{n}\right\}$ is a cozero refinement of $\left\{U_{n}\right\}$. That $X$ is a $c b$-space, now follows from Theorem 1 .

Added in proof. A locally compact, countably paracompact space need not be a $c b$-space. See the example at the end of $\$ 3$ in [8].

\section{REFERENCES}

1. C. H. Dowker, On countably paracompact spaces, Canad. J. Math. 3 (1951), 219-224.

2. J. G. Horne, Jr., Countable paracompactness and cb-spaces, Notices Amer. Math. Soc. 6 (1959), 629-630.

3. L. Gillman and M. Jerison, Rings of continuous functions, Van Nostrand, Princeton, N. J., 1960.

4. F. Ishikawa, On countably paracompact spaces, Proc. Japan Acad. 31 (1955), 686-687.

5. M. J. Mansfield, On countably paracompact normal spaces, Canad. J. Math. 9 (1957), 443-449. 236.

6. K. Morita, Paracompactness and product spaces, Fund. Math. 50 (1961), 223-

7. T. Ishii, Some characterizations of m-paracompact spaces. I, Proc. Japan Acad. 38 (1962), 480-483.

8. J. E. Mack and D. G. Johnson, The Dedekind completion of $C(X)$, Pacific J. Math. (to appear).

OHIo University 